\title{
Study on Urban and Rural Ecotone Vehicles Acceleration Interference Model
}

Li Yan

Management Department of Heilongjiang Institute of

Technology

Harbin, China

\begin{abstract}
In this paper, the status quo of urban and rural ecotone road accident-prone, through the analysis of changes in vehicles acceleration feature, choice of three-direction acceleration interference as road safety indicators. Threedirection acceleration interference models are established based on acceleration interference theory. To select urban and rural ecotone typical sections, to measure vehicle acceleration changes under different road conditions, to conclude the main rule of traffic accidents, and to verify the validity of the acceleration model.
\end{abstract}

Index Terms - Urban and rural ecotone, three-direction acceleration, discretization of the model, acceleration interference model.

\section{INTRODUCTION}

Urban and rural ecotone is the combined the binary zone [1]. Because of more type vehicles, more serious pedestrian and vehicle mixed traffic, leading to urban and rural ecotone bad road traffic environment [2]. During vehicles running, speed change is more complex and more fluctuation, especially vehicle acceleration change has an important impact on traffic safety. In urban and rural ecotone section, the vehicle will be affected by the road alignment, road condition and traffic flow, and drivers tend to be more frequent braking or acceleration, in order to keep the continuity of the vehicle running speed. The running speed may change at any time, frequent acceleration and deceleration of the case [3]. Intensity of vehicle speed change, i.e., the acceleration is more objective indicator of the changes of vehicle traveling state [4]. Therefore, speed fluctuation of vehicles can be represented by acceleration standard deviation [5], see the Equation (1).

$$
\left\{\begin{array}{l}
\sigma=\left\{\left(\frac{1}{T}\right) \int_{0}^{T}[a(t)-\bar{a}]^{2} d t\right\}^{\frac{1}{2}} \\
\bar{a}=\left(\frac{1}{T}\right) \int_{0}^{T} a(t) d t
\end{array}\right.
$$

Where: $\sigma$-acceleration interference, $\mathrm{m} / \mathrm{s}^{2}, \mathrm{~T}$ - the total running time of the vehicles, $\mathrm{s}, a(t)-\mathrm{T}$ moment acceleration, $\mathrm{m} / \mathrm{s}^{2}, \bar{a}$ - average acceleration, $\mathrm{m} / \mathrm{s}^{2}$.

\author{
Li Xiansheng \\ Transportation and Traffic College of Jilin University \\ Changchun, China
}

\section{URBAN AND RURAL ECOTONE THREE-DIRECTION ACCELERATION INTERFERENCE MODEL}

\section{A. Lateral Acceleration Interference Model}

When vehicles traveling along the road horizontal curve, by centrifugal force and centripetal force. In order to improve the stability of the vehicles, set the ultra-high angle horizontal curve in the road at the general, to overcome the centrifugal force generated when the vehicle is cornering.

Centripetal force and centrifugal force:

$$
\begin{aligned}
& F_{n}=m g \tan \alpha \approx m g \sin \alpha \\
& F_{r}=\frac{m v^{2}}{R}
\end{aligned}
$$

Where: m-car quality, g-acceleration of gravity, $\alpha$ - the road is ultra-high tilt angle, $\mathrm{R}$-flat curve radius.

By the Equation (2) and the Equation (3) the lateral force of the vehicle suffered:

$$
F_{h}=F_{r} \cos \alpha-F_{n}=F_{r} \cos \alpha-m g \sin \alpha
$$

Where: $\sin \alpha=\frac{h_{c}}{\sqrt{b^{2}+h_{c}^{2}}}, \quad \cos \alpha=\frac{b}{\sqrt{b^{2}+h_{c}^{2}}}$, $h_{c}$ is an elevation value, $\mathrm{b}$ is on the curve as a curve somewhere on the road width half, into the Equation (1) can be obtained:

$$
a_{h}=\left(\frac{V^{2}(t)}{R}-\frac{g}{b}\right) \frac{b}{\sqrt{b^{2}+h_{c}^{2}}}
$$

Lateral acceleration interference model by the Equation (5) and the Equation (1) can be obtained:

$$
\left\{\begin{array}{l}
\sigma_{h}=\left\{\left(\frac{1}{T}\right) \int_{0}^{T}\left[\left(\frac{V^{2}(t)}{R}-\frac{g}{b}\right) \frac{b}{\sqrt{b^{2}+h_{c}^{2}}}-\bar{a}\right]^{2} d t\right\}^{\frac{1}{2}} \\
\bar{a}=\frac{1}{T} \int_{0}^{T} a(t) d t=\frac{1}{T} \int_{0}^{T}\left(\frac{V^{2}(t)}{R}-\frac{g}{b}\right) \frac{b}{\sqrt{b^{2}+h_{c}^{2}}} d t
\end{array}\right.
$$

\section{B. Axial Acceleration Interference Model}

Axial acceleration is generated in the axial acceleration and deceleration of the traveling vehicle, the axial acceleration direction with the vehicle traveling direction, the acceleration 
size depends on the dynamic performance of the vehicle and the running resistance can be expressed as:

$$
a_{z}=\frac{\lambda g}{\delta}(D-f-i)
$$

Where: $\lambda$ - altitude load correction factor, $\delta$ - inertia force coefficient, $D$ - automotive power coefficient, $f$ rolling resistance coefficient, $i$ - vertical slope.

$$
i=\frac{d y}{d x}=i_{1} \pm \frac{l}{R_{s}}
$$

The power coefficient $D$ as:

$$
\left\{\begin{array}{l}
D=\frac{K T_{0}-K^{\prime} V^{2}(t)}{G} \\
K=\frac{i_{g} i_{0} \eta_{T}}{r} \\
K^{\prime}=\frac{C_{D} A}{21.15}
\end{array}\right.
$$

Where: $i_{g}$ - the vehicle transmission speed ratio, $i_{0}-$ the main reduction gear rotation ratio, $\eta_{T}$ - inertia coefficient, $r$ wheel radius, $C_{D}$ - air resistance coefficient, $T_{0}$ - loading rate, the paper take $100 \%, A$ - vehicles traveling direction of the projection area.

By the Equation (7), the Equation (8) and the Equation (9) known, the axial direction of the straight slope segment acceleration is:

$$
a_{z}=\frac{\lambda g}{\delta}\left(\frac{K T_{0}-K^{\prime} V^{2}(t)}{G}-f-i\right)
$$

By the Equation (10) and the Equation (1) axial acceleration interference model is:

$$
\left\{\begin{array}{l}
\sigma_{z}=\left\{\left(\frac{1}{T}\right) \int_{0}^{T}\left[\frac{\lambda g}{\delta}\left(\frac{K T_{0}-K^{\prime} V^{2}(t)}{G}-f-i\right)-\bar{a}\right]^{2} d t\right\}^{\frac{1}{2}} \\
\bar{a}=\frac{1}{T} \int_{0}^{T} a(t) d t=\frac{1}{T} \int_{0}^{T} \frac{\lambda g}{\delta}\left(\frac{K T_{0}-K^{\prime} V^{2}(t)}{G}-f-i\right) d t
\end{array}\right.
$$

\section{Vertical Acceleration Interference Model}

Vehicles traveling on a vertical curve generate the vertical centrifugal force, which produce vertical acceleration. This is due to the vertical section on the vertical curve caused, different vertical curve radius different vertical acceleration, namely:

$$
a_{s}=\frac{V^{2}(t)}{3.6^{2} R_{s}}=\frac{V^{2}(t)}{12.96 R_{s}}
$$

Where: $R_{s}$ - radius of curvature at the largest radius of curvature, $\mathrm{m}$.

Can be obtained by the Equation (12) and the Equation (1) vertical acceleration interference model is:

$$
\left\{\begin{array}{l}
\sigma_{s}=\left\{\frac{1}{T} \int_{0}^{T}\left[\frac{V^{2}(t)}{12.96 R_{s}}-\bar{a}\right]^{2} d t\right\}^{\frac{1}{2}} \\
\bar{a}=\frac{1}{T} \int_{0}^{T} a(t) d t=\frac{1}{T} \int_{0}^{T} \frac{V^{2}(t)}{12.96 R_{s}} d t
\end{array}\right.
$$

\section{Model Discretized}

Using equipment to consecutive time intervals ( $\Delta t)$ detecting the vehicle running acceleration, acceleration interference model is:

$$
\sigma=\left\{\left(\frac{1}{T}\right) \sum\left[a_{i}-\bar{a}\right]^{2} \Delta t\right\}^{\frac{1}{2}}
$$

Where: $\mathrm{T}$ is the observation time, $a_{i}$ for time acceleration, $\bar{a}$ average acceleration, $\Delta t$ time equal-length sampling.

Of $\sum\left[a_{i}-\bar{a}\right]^{2} \Delta t$ transformation:

$\sum\left[a_{i}-\bar{a}\right]^{2} \Delta t=\sum a_{i}^{2} \Delta t-\sum 2 a_{i} \bar{a} \Delta t+\sum \bar{a}^{2} \Delta t=\sum a_{i}^{2} \Delta t-2 \bar{a}^{2} T+\bar{a}^{2} T=\sum a_{i}^{2} \Delta t+\bar{a}^{2} T$

Substituting acceleration interference Equation is:

$$
\sigma=\left\{\left(\frac{1}{T}\right) \sum a_{i}^{2} \Delta t-\bar{a}^{2}\right\}^{\frac{1}{2}}=\left\{\frac{\Delta t(T-\Delta t)}{T^{2}} \sum a_{i}^{2}\right\}^{\frac{1}{2}}
$$

The Equation (15) into the Equation (6), the Equation (11) and the Equation (13) obtained:

$$
\sigma=\left\{\begin{array}{l}
\left\{\frac{\Delta t(T-\Delta t)}{T^{2}} \sum\left[\left(\frac{V^{2}(t)}{R}-\frac{g}{b}\right) \frac{b}{\sqrt{b^{2}+h_{c}^{2}}}\right]^{2}\right. \\
\left\{\frac{\Delta t(T-\Delta t)}{T^{2}} \sum\left[\frac{\lambda g}{\delta}\left(\frac{K T_{0}-K^{\prime} V^{2}(t)}{G}-f-i\right)\right]^{2}\right\}^{\frac{1}{2}} \\
\left\{\frac{\Delta t(T-\Delta t)}{T^{2}} \sum\left[\frac{V^{2}(t)}{12.96 R_{s}}\right]^{2}\right\}^{\frac{1}{2}}
\end{array}\right.
$$

\section{MEASURED ACCELERATION ANALYSIS}

Test sections selected in Harbin, two sections of urban and rural ecotone. First experimental section for isolation fence section, second experimental section for un-isolation fence section.During the test, the vehicle is running possible speed. Good road condition means the road surface is clean, smooth, and not consider the snow, icing and snowfall, good traffic condition is free stream running state, does not consider cross, pedestrians and non-motorized interference, the driver who is skilled is able to skillfully driving a vehicle is in good health, tend to travel at higher speeds, but not risky. Consider security reasons, the principles of the above possible speed corresponding prediction model calculation, under certain conditions, can be obtained by actual measurement. This paper refers to possible speed to meet the basic road condition, traffic condition, and driver quality premise vehicle maximum safe running speed.

The experimental data of the vehicle traveling in the $1 \mathrm{st}$ section is shown in Fig. 1,Fig.2,Fig.3. 


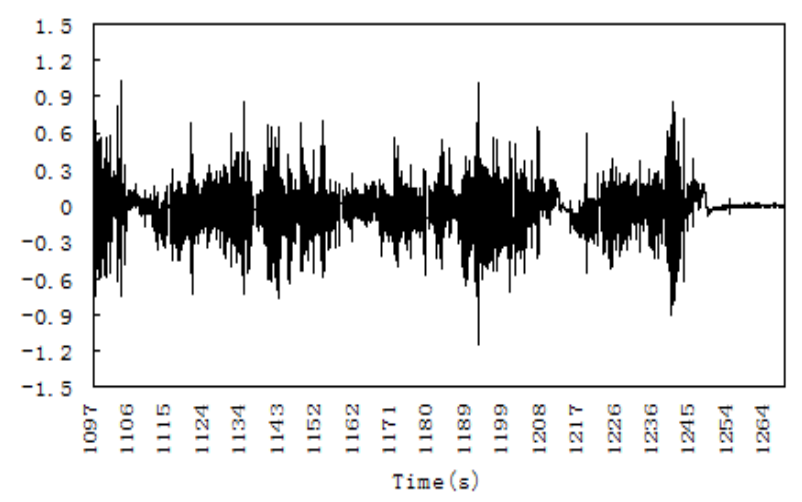

Fig.1 Longitudinal acceleration

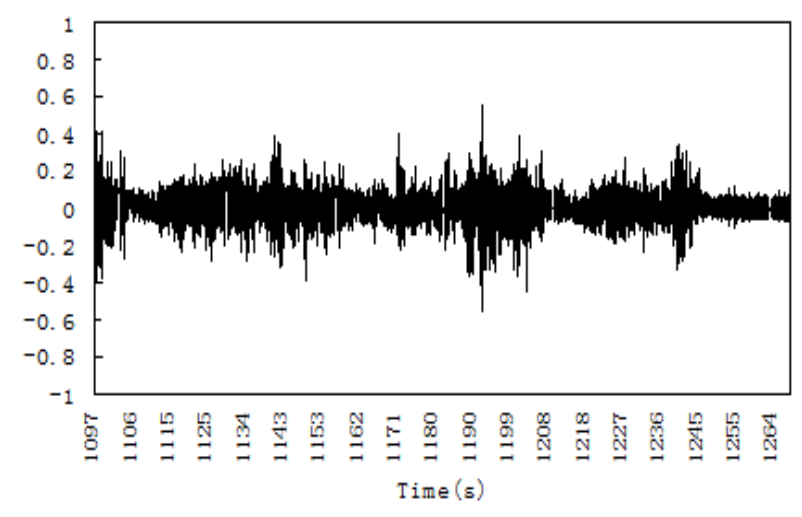

Fig.2 Lateral acceleration

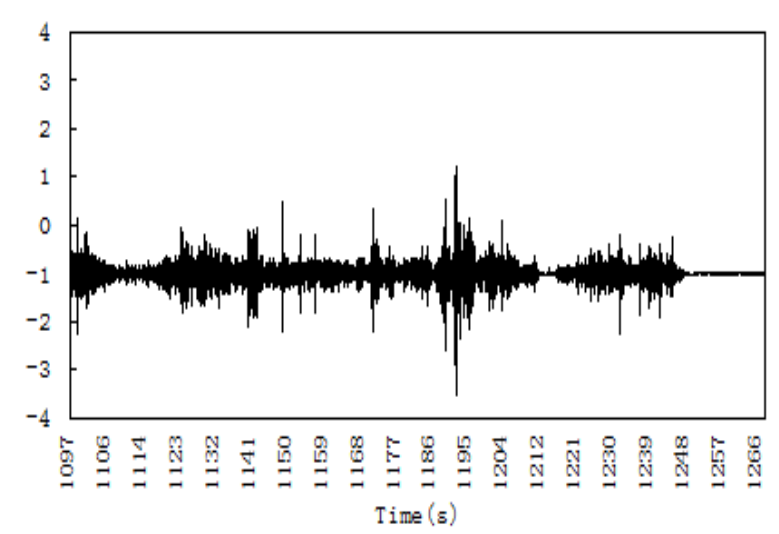

Fig.3 Vertical acceleration

The experimental data of the vehicle traveling in the 2 nd section is shown in Fig. 4, Fig .5, Fig .6.

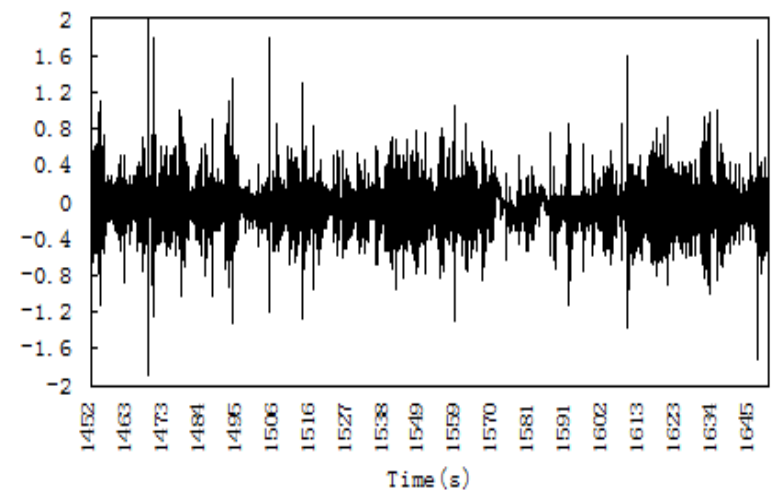

Fig. 4 Longitudinal acceleration

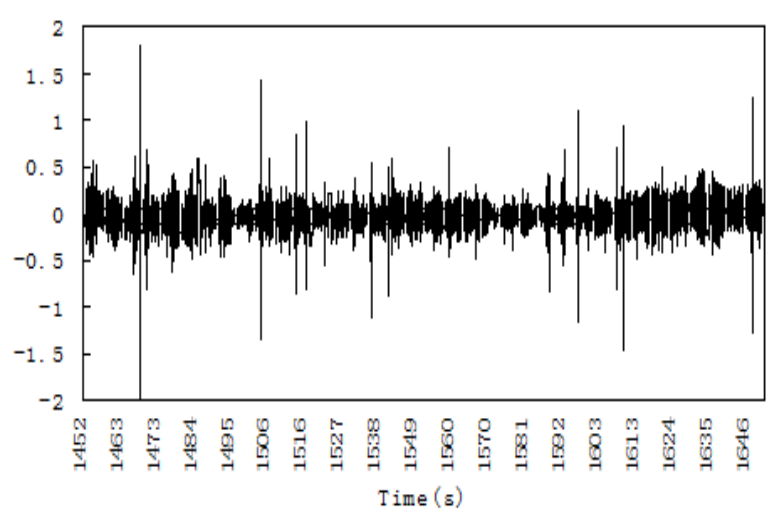

Fig.5 Lateral acceleration

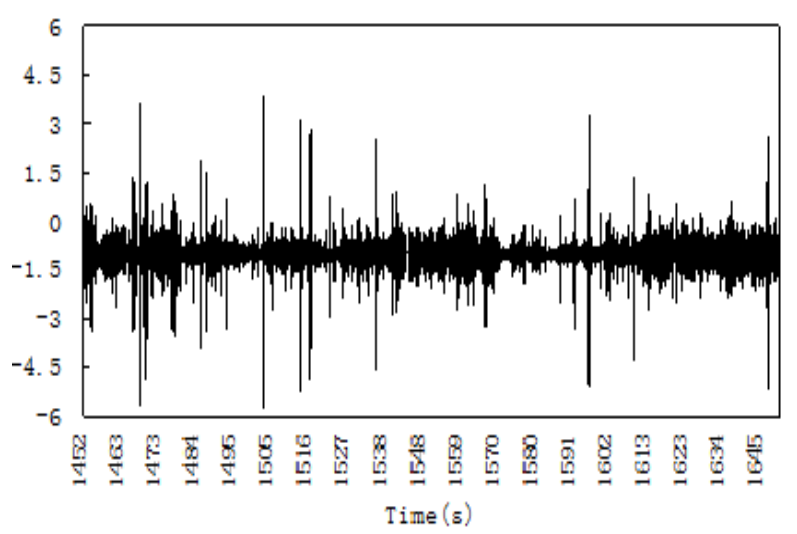

Fig.6 Vertical acceleration

\section{CONCLUSION}

Use of urban and rural ecotone roads measured experimental data, the combination of these models can be calculated in the urban and rural ecotone with the changes in the characteristics of the different sections of the vehicle in speed. Through analysis of two types of urban and rural ecotone respectively measured the resulting data, found that the types of road traffic accidents are significant difference in different urban and rural ecotone sections. On the isolation fence section, vehicles lateral acceleration larger, vehicles scraping a high proportion of traffic accidents, without the 
isolation fence section, vehicles longitudinal acceleration larger, vehicles rear-end accidents a high proportion.

\section{REFERENCES}

[1] Chen Youqi. "Urban and rural ecotone name debate". Beijing: Geography and Territorial Research, 1995(2), pp.47-53.

[2] Li Yan, Li Xiansheng. "Traffic safety evaluation in the ruralurban continuum based on ANP". 2009 Second International Conference on Intelligent Computation Technology and Automation, Hunan province, pp.368-372, October 2009.
[3] Liu Tangzhi, Tang Boming, Ling Jianming, Liu Li. "Urban fringe of Road Traffic Accidents in law analysis and Countermeasures". Chongqing: Chongqing Jiaotong University (Natural Science), 2008(27), pp.767-783.

[4] Li Lin, Xu Jianmin, Ye Fan. "Based on the road plane linear acceleration interference model". Beijing: Journal of China and foreign highway, 2010, 30(2), pp.18-21.

[5] Xu Lunhui, Zhou Jing. "Based on road structure acceleration interference model and comfort evaluation". Beijing: Highway, 2007(3),pp.102-106. 